\title{
A New Particle Filter Target Tracking Algorithm Based on Genetic Algorithm
}

\author{
Wan-li Zhang* and Xiao-ying Yang
}

College of Information Engineering, Suzhou University, Suzhou, 234000, P.R. China

\begin{abstract}
Aimed to problem that particles exist degradation in particle filter (PF) algorithm for target tracking which is used in Wireless Sensor Networks, a new particle filter target tracking algorithm (GPF) based on genetic algorithm was proposed in this paper. First, based on the description of the PF algorithm, the factors that affect the degradation were analyzed, which were importance function and re-sampling technique. Then, a new importance function which can make the current measured value take effect was constructed and the re-sampling technique was mended by using genetic algorithms in the GPF. Finally, the simulation tracking results showed that the GPF algorithm has better tracking accuracy than that of PF.
\end{abstract}

Keywords: Genetic algorithm, particle filter, target tracking, wireless sensor networks.

\section{INTRODUCTION}

Target tracking model of Wireless Sensor Networks (WSN) usually is nonlinear and non-Gaussian model $[1,2]$. $\mathrm{PF}$ algorithm is a filtering method that based on a sequential Monte Carlo [3]. As it attached to approximate the posterior probability distribution by using a series of random particles with weights, it does not subject to non-linear, non-Gaussian constraints. Thus, it has been widely used in target tracking in WSN [4], fault diagnosis [5], economic forecasting [6] and other fields. However, the algorithm has a serious degradation [7], thereby affecting the tracking effect. PF algorithm is improved in Literature [8-10]. In this paper, based on PF algorithm, GPF was proposed which is suitable for wireless sensor networks.

\section{BASIC PF ALGORITHM}

\subsection{PF Algorithm}

Algorithm flow chart is shown in Fig. (1).

According to the algorithm flow chart, step of particle filter as follows:

1) Initialize. $\mathrm{N}$ point set of particles $\left\{x_{0}^{i}, w_{0}^{i}\right\}_{i=1}^{N}$ are obtained by initializing probability density distribution $p\left(x_{0}\right)$. Initialize the weights of all the particles as $w_{0}^{i}=1 / N \mathrm{X}$;

2) Particle state predict. At time $k(k=1,2,3 \ldots)$, predicted particle state at time $k$ through the movement of objects

*Address correspondence to this author at the College of Information Engineering, Suzhou University, Suzhou, 234000, P.R. China;

Tel: 13866547730; E-mail: zhangwanli0557@aliyun.com kinetic model $P\left(x_{k} \mid x_{k-1}=x_{k-1}^{i}\right)$, produce set of particles at $k$ moment, particle weights are $w_{k}^{i}=1 / N$.

3) Update the particle. Using equation (1) to update weights of each particle in particle set at time $k$.

$w_{k}^{i}=w_{k-1}^{i} \frac{p\left(z_{k} \mid x_{k}^{i}\right) p\left(x_{k}^{i} \mid x_{k-1}^{i}\right)}{q\left(x_{k}^{i} \mid x_{k}, z_{k}\right)}$

4) Re-sample. Define valid sampling scale as $N_{e f f}=1 / \sum_{i-1}^{N} w_{i}$. If $N_{e f f}<N_{t h},\left(N_{t h}\right.$ is a threshold $)$ then resampled.

\subsection{Particle Degradation}

Particle degradation is a inevitable phenomenon in PF algorithm. Iteration of the loop, in addition to a particle, the other particle weights is negligible [11]. An important technology of reducing the particle degradation is selection of importance functions and re-sampling [12].

1) The importance function

Importance function affects the efficiency of the algorithm and the degradation rate of weight coefficients. Make importance functions easy to sample and minimum variance weights are the two principles of choosing importance function. Importance function should be as close as possible to the system status posterior probability, which can reduce the variance of the importance weights and make variance of its weights the smallest.

\section{2) Re-sample}

Re-sampling is another factor affecting to reduce particle degradation. The aim is to leave a large particle weights, 


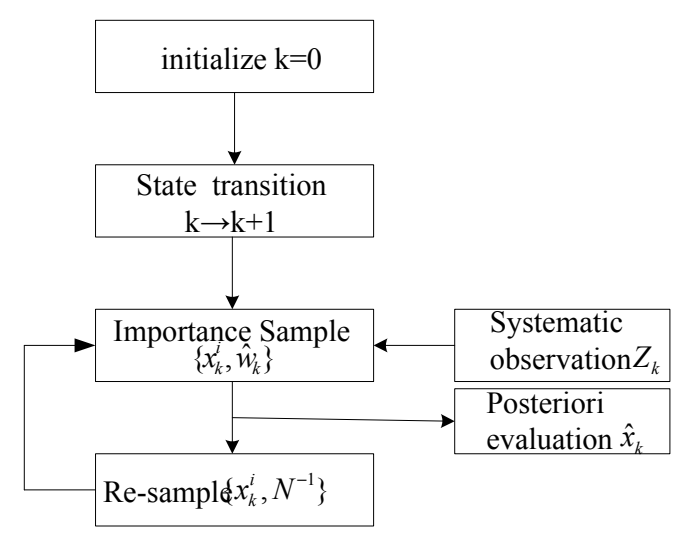

Fig. (1). PF algorithm flow chart.

reduce the number of particles with smaller weights. When reduce to a threshold, namely, the apparent degradation appear, re-sample the existing set of samples and generate a new set of samples.

\section{GPF ALGORITHM}

\subsection{Mend the Importance Function}

In order to reduce the particle degradation mitigation in the PF, first, mend the importance function of PF. Select importance function that can make the current measured value function [13]. Importance function is approximation of the posterior distribution, when the likelihood function compared with the transition probability distribution is too concentrated, closer the posterior density than prior density. Therefore, on the basis of the likelihood function using a new approximation, can play new tracking results.

Construction of importance function:

Order $m_{k}=\left(x_{k}\right)^{2}, \quad p\left(m_{k} / z_{k}\right)$ is the prior distribution probability density function of $m_{k}$, repeat sampled from $p\left(m_{k} / z_{k}\right) \quad$ until $m_{k} \geq 0 \cdot m_{k}$ is recorded as $m_{k}^{i} \sim p\left(m_{k} / z_{k}\right)$. Using $p\left(x_{k} / m_{k}^{i}\right)$ to construct importance function, at this point, $x_{k}^{i}$ depends only on $z_{k}$, nothing to do with $x_{k-1}^{i}$. Thus launched $q\left(x_{k}^{i} / x_{k-1}^{i}, z_{k}\right)=$ $p\left(x_{k}^{i} / m_{k}^{i}\right) p\left(x_{k}^{i} / z_{k}\right)$ 。

Seeking $\frac{p\left(x_{k}^{i} / z_{k}\right)}{p\left(m_{k}^{i} / z_{k}\right)}$ according weight update equation $w_{k}^{i} \propto w_{k-1}^{i} p\left(x_{k}^{i} / x_{k-1}^{i}\right) \frac{p\left(x_{k}^{i} / z_{k}\right)}{p\left(m_{k}^{i} / z_{k}\right)}$. Its true value may be considered as proportional. $p\left(x_{k} / z_{k}\right)$ and $p\left(m_{k} / z_{k}\right)$, respectively, quadrature for $x_{k}$ and $m_{k}$. Between the ratio of the probability density is proportional to $\left|\frac{d m_{k}}{d x_{k}}\right|=2 x_{k}$. At this time, weight update equation is shown in equation (2).

$$
\begin{aligned}
& w_{k}^{i} \propto w_{k-1}^{i} p\left(x_{k}^{i} / x_{k-1}^{i}\right) \frac{p\left(x_{k}^{i} / z_{k}\right)}{p\left(m_{k}^{i} / z_{k}\right)} \\
& \propto w_{k-1}^{i} p\left(x_{k}^{i} / x_{k-1}^{i}\right) x_{k}^{i}
\end{aligned}
$$

So after sampling, a new importance function is obtained.

\subsection{Mend Re-sample by Using Genetic Algorithm}

First, according to the weight of the particle, $N$ new particles are obtained from the old set of $\left\{x_{k}^{i}, w_{k}^{i}\right\}_{i=1}^{N}$ by roulette approach with the select probability of $p_{s}$.

Secondly, randomly selects two correspond articles from the new set of particles $\left\{\hat{x}_{k}^{i}, \hat{w}_{k}^{i}\right\}_{i=1}^{N_{s}}$ and the old set of particles, $\left\{x_{k}^{i}, w_{k}^{i}\right\}_{i=1}^{N}$, With probability $p_{c}$ cross processing, resulting in producing two new particles $\hat{x}_{k}^{i^{\prime}}, \hat{x}_{k}^{i^{\prime \prime}}$, alternatively replaced $\hat{x}_{k}^{i}$, get a new set of particles $\left\{\hat{x}_{k}^{i}, \hat{w}_{k}^{i}\right\}_{i=1}^{N^{\prime}}$, crossover operation generates $N_{c}=N^{*} p_{c}$ new particles.

Finally, particles $\left\{\hat{x}_{k}^{i}, \hat{w}_{k}^{i}\right\}_{i=1}^{N^{\prime}}$ which are obtained through selection and crossover are mutated with mutation probability $p_{m}$. Randomly select a particle $\hat{x}_{k}^{i}$ from particles set $\left\{\hat{x}_{k}^{i}, \hat{w}_{k}^{i}\right\}_{i=1}^{N^{\prime}}$ to do mutation operation to get a new particle $\hat{x}_{k}^{i^{\prime}}$, With $\hat{x}_{k}^{i^{\prime}}$ replace $\hat{\boldsymbol{x}}_{\boldsymbol{k}}^{i}$, get new particle set $\left\{\hat{x}_{k}^{i}, \hat{w}_{k}^{i}\right\}_{i=1}^{N^{\prime \prime}}$, mutation operation generates $N_{m}=N^{*} p_{m}$ new particles.

As randomly selected rule make the same probability of each particle being selected, increasing the particle crossover, mutation opportunities to mend poor particle problem. Cross is concentrated in while selecting both the old and new particles, not only the good genes to be retained, but also mends the efficiency of the cross.

\subsection{Steps of GPF Algorithm}

1) Initialization. N-point set of particles $\left\{x_{0}^{i}, w_{0}^{i}\right\}_{i=1}^{N}$ are obtained by initial probability density distribution $p\left(x_{0}\right)$. Initialize the weight of all the particles is $w_{0}^{i}=1 / N$;

2) Particle state prediction. At time $k(k=1,2,3 \ldots)$, predict particle state at time $k$ by dynamic model $P\left(x_{k} \mid x_{k-1}=x_{k-1}^{i}\right)$ of the movement of objects.

3) Particle weight. Select importance density as forward probability function of the target motion. The update formula show as equation (3).

$$
\begin{aligned}
& w_{k}^{i} \propto w_{k-1}^{i} p\left(x_{k}^{i} / x_{k-1}^{i}\right) \frac{p\left(x_{k}^{i} / z_{k}\right)}{p\left(m_{k}^{i} / z_{k}\right)} \\
& \propto w_{k-1}^{i} p\left(x_{k}^{i} / x_{k-1}^{i}\right) x_{k}^{i}
\end{aligned}
$$




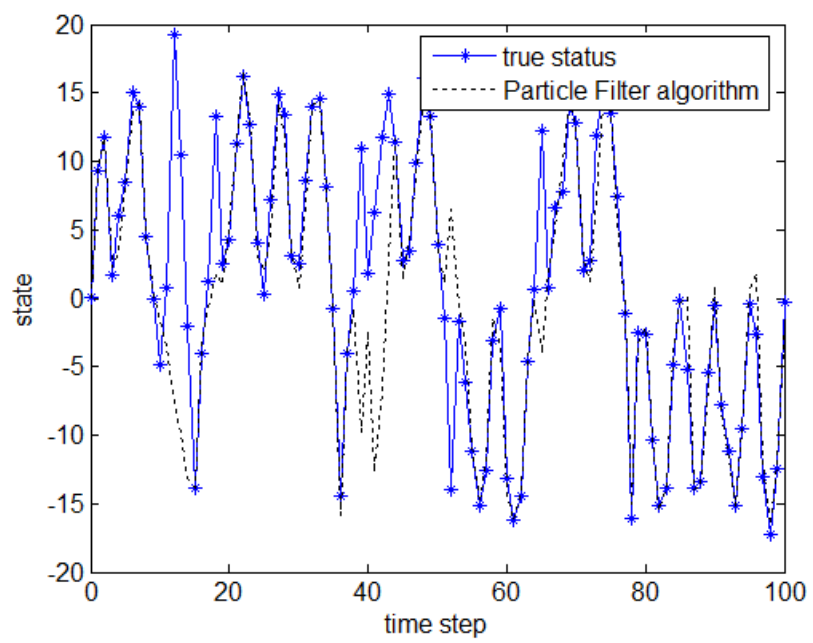

Fig. (2). Tracking simulation diagram of PF.

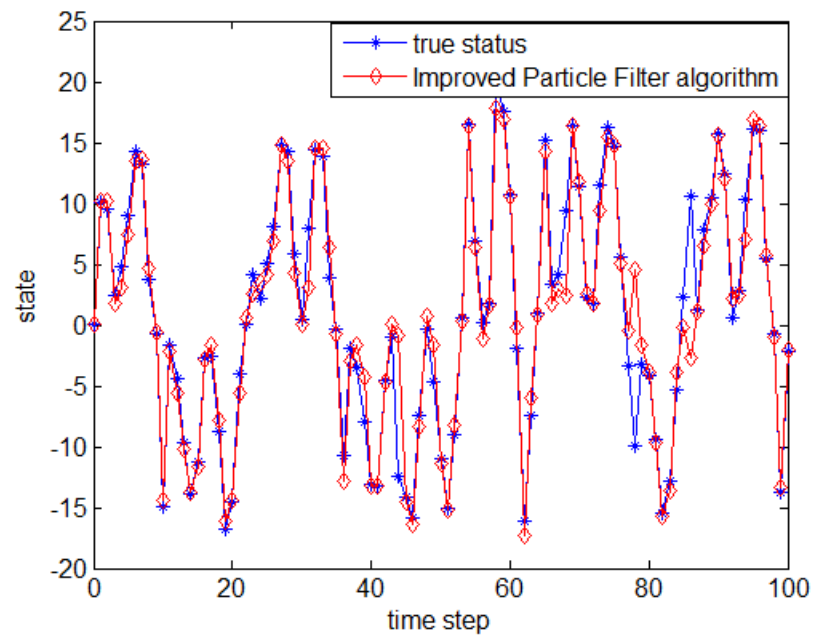

Fig. (3). Tracking chart of GPF algorithm.

4) Genetic manipulation of particles. Use genetic algorithm to re sample the set of particles which produce in the step 3). $N$ particles with maximum weight are selected from the results $\left\{\hat{x}_{k}^{i}, \hat{w}_{k}^{i}\right\}_{i=1}^{N "}$ of crossover and mutation. Then go to Step (2), for the next forecast.

\section{SIMULATION AND ANALYSIS OF ALGORITHMS}

The simulation uses the used system equations to experiment, show as the equation (4).

$\left\{\begin{array}{l}x_{k}=f\left(x_{k-1}, k\right)+u_{k-1} \\ z_{k}=x_{k}^{2} / 20+v_{k}\end{array}\right.$

Among

$f\left(x_{k-1}, k\right)=x_{k-1} / 2+25 x_{k-1} /\left(1+x_{k-1}^{2}\right)+8 \cos (1.2 k)$, both $u_{k-1}$

and $v_{k}$ is a mean-variance matrix, respectively, is zero-mean

Gaussian random variables of $Q_{K-1}$ and $R_{K} \cdot Q_{K-1}=R_{k}=1$, $T=100$.
Elementary particle filter algorithm and the algorithm proposed in this paper use the above nonlinear model of moving target tracking to simulate, and simulate of the root mean square error between several algorithms. Root mean square error is defined by using equation (5).

$$
R M S=\sqrt{\sum_{i=1}^{N} \frac{1}{T}\left(\hat{x}_{k}-x_{k}\right)^{2}}
$$

Where, $N$ is the number of simulations and the value is $100 ; T$ is the sampling period; $\hat{x}_{k}$ and $x_{k}$ respectively represent algorithms tracking results and system true status. The simulation results are shown in Fig. (2) and Fig. (3).

Fig. (2) shows the tracking simulation diagram of the basic $P F$ algorithm. It can be seen from the figure that the basic PF algorithm can approximate the real results when it tracks moving targets, but a great error in some sampling points.

Fig. (3) shows tracking simulation diagram of the GPF algorithm. It can be seen from the simulation results that the 
Table 1. Comparison of RMSE of two algorithms.

\begin{tabular}{|c|c|c|}
\hline \multirow{2}{*}{} & \multicolumn{2}{|c|}{ Algorithm } \\
\cline { 2 - 3 } & PF Algorithm & GPF Algorithm \\
\hline \hline error & 4.9782 & 2.7063 \\
\hline
\end{tabular}

GPF algorithm can better approximate the real result than the basic PF and a small error in some sampling points. Table 1 shows the comparison of tracking error of the two algorithms. As the error parameters of the system equations are random variables, the error values are not a determined value, but still it is clear that the advantages of GPF.

\section{CONCLUSION}

PF target tracking algorithm used in wireless sensor networks was researched in this paper. An new particle filter tracking algorithm (GPF) was proposed to solve particle degradation problem. GPF algorithm re-constructed the importance of the function and mended re-sample with genetic algorithm. Finally the simulation showed the new particle filter algorithm had a new tracking effect.

\section{ABOUT THE AUTHORS}

First Author Wanli-Zhang was born in 1977. He received the master degree of computer technology from Anhui University in 2009. He is a lecturer at College of Information Engineering, Suzhou University, China. He research interests include Wireless sensor networks, Internet of Things and computer network.

Second Author Xiaoying-Yang was born in 1983. She received the master degree of computer technology from Guilin University of Electronic Technology in 2010. She is a lecturer at College of Information Engineering, Suzhou University, China. She research interests include Wireless sensor networks and database system.

\section{CONFLICT OF INTEREST}

The authors confirm that this article content has no conflict of interest.

\section{ACKNOWLEDGEMENTS}

This work was supported by Young Talents Fund Project in Anhui Province of China (No. 2013SQRL083ZD), Natural Science Research Project in Anhui Province of China (No. KJ2014A247, No. KJ2014ZD31) and Students innovation training project (No. 201310379019).

\section{REFERENCES}

[1] X. Huang, Y. Wang, and B. Chen, "Distributed target tracking algorithm based on particle filtering," Computer Engineering, vol. 37-4, pp. 84-86, 2011.

[2] D. Djuric, "Particle filters," IEEE Signal Processing Magazine, vol. 10-2, pp. 19-38, 2003

[3] X. Kuang, H. Huo, Y. Liu, Y. Wu, and H. Zhang, "Study of the target tracking based on pf algorithm in wireless sensor networks," Chinese Journal of Sensors and Actuators, vol. 22-7, pp. 10291033, 2009.

[4] Doucet, S. Godsill, and C. Andrieu, "A survey of convergence results on particle filtering methods for practitioners," IEEE Transactions on Signal Processing, vol. 50-2, pp. 736-746, 2002.

[5] Y. Ruan, P. Willett, A. Marrs, F. Palmieri, and S. Marano, "Practical fusion of quantized measurements via particle filtering," IEEE Transactions Aerospace and Electronic Systems, vol. 44-1, pp. 1529, 2008.

[6] A.C. Sankaranarayanan, A. Srivastava, and R. Chellappa., "Algorithmic and architectural optimizations for computationally efficient particle filtering," IEEE Transactions Image Processing, vol. 17-5, pp. 737-748, 2008

[7] J. Wang, L. Ye, and Q. Zhang, "Genetic particle filter and its application to motion tracking," Journal of Beijing Broadcasting Institute (Science and Technology), vol. 112-12, pp. 23-28, 2005.

[8] J. Meng, J. Liu, J. Wang, and M. Han, "Target tracking based on optimized particle filter algorithm," Journal of Software, vol. 8-5, pp. 1140-1144, 2013.

[9] M. Li, B. Pang, Y. He and F. Nian, "Particle filter new by genetic algorithm and particle swarm optimization algorithm," Journal of Software, vol. 8-3, pp. 666-672, 2013.

[10] S. Li, L. Sun, X. Li, and D. Wang, "Research on a new terrain aided positioning model," Journal of Software, vol. 6-5, pp. $937-$ 942, 2011.

[11] X. Qiu, F. Miao, and Q. Liu, "Genetic particle filtering algorithm applied in wireless sensor networks," Journal of Nanjing Normal University (Engineering And Technology Edition), vol. 10-3, pp. 231-234, 2010.

[12] N. Yang, F. Qian, and R. Zhu, "New particle filter based on genetic algorithm," Journal of Shanghai Jiaotong University, vol. 45-10, pp. 98-102, 2011.

[13] J. Zheng, "New particle filtering algorithm for target tracking in WMSN," Computer Engineering and Applications, vol. 47-15, pp. 83-85, 2011. 